\title{
Approximate Solution of Schrodinger Equation for Modified Poschl-Teller plus Trigonometric Rosen-Morse Non-Central Potentials in Terms of Finite Romanovski Polynomials
}

\author{
A. Suparmi ${ }^{1}$, C. Cari $^{2}$, J. Handhika $^{3}$, C. Yanuarief $^{4}$, H. Marini ${ }^{5}$ \\ ${ }^{1,2}$ (Physics Department, SebelasMaret University, Indonesia) \\ ${ }^{3,4,5}$ (Graduate Student of Physics Department, SebelasMaret University, Indonesia)
}

\begin{abstract}
The energy eigenvalues and eigenfunctions of Schrodinger equation for Modified Poschl-Teller potential plus trigonometric Rosen-Morse non-central potential are investigated approximately in terms of finite Romanovski polynomial. The approximation has been made to solve the radial Schrodinger equation. The approximate bound state energy eigenvalues are given in a closed form and corresponding radial and eigenfunctions are obtained in terms of Romanovski polynomials. The polar eigenfunctions are obtained in terms of Romanovski polynomials. The trigonometric Rosen-Morse potential is considered to be perturbation factor to the modified Poschl-Teller potential since it causes the decrease of the length of angular momentum vectors.
\end{abstract}

Keywords: Approximate Solution, Schrodinger Equation, Modified Poschl-Teller Potential, Trigonometric Rosen Morse Non-Central Potential, FiniteRomanovski Polynomials

\section{Introduction}

The exact analytical solutions of Schrodinger equations for some physical potentials are very essential since the knowledge of wavefunctions and energy contains all possible important information of the physical properties of quantum system. Recently, conciderably efforts have been paid to obtain the exact solution of the central and non-central potentials. There are only a few potentials for which the Schrodinger equation can be solved exactly. In general, one has to resort to numerical techniques or approximation schemes. For many quantum mechanical systems, most approximation methods used are shifted $1 / N$ expansion, WKB method, perturbation method, supersymmetric quantum mechanics and idea of shape invariance, etc. Although some methods produce eigenvalues easily but give complicated eigenfunctions.

In recent years, numerous studies have been made in analyzing the bound state of charged particle moving in a vector potential and a non-central scalar potential, such as an electron moving in a Coulomb field with simultaneously precence of Aharonov-Bohm field [1,2], or/and magnetic monopole [3], Makarov potential [4] or ring-shaped-oscillator potential [5-8], etc. In most of these studies, the eigenvalues and eigenfunctions are obtained using separation variables in spherical coordinate system. Very recently, supersymmetric quantum mechanics with the idea of shape invariance [9], factorization method [10-11], and Nikiforov-Uvarov method [12] are widely used to derive the energy spectrum and the wave function of a charge particle moving in noncentral potential.

Very recently, an alternative method called as finite Romanovski polynomials, which is a traditional method, consist of reducing Schrodinger equation by an appropriate change of the variable to that of very form of generalized hypergeometric equation[12-13]. The polynomial was discovered by Sir E.J.Routhand rediscovered 45 years later by V. I. Romanovski $[14,15]$. The notion "finite" refers to the observation that, for any given set of parameters (i.e. in any potential) only a finite of polynomials appear orthogonal

It is known that for very limited potential, the three dimansional radial Schrodinger equation is exactly solvable only for s-wave $(l=0)$. However, the three dimensional radial Schrodinger equation for the spherically symmetric potentials can not be solved analytically for $l \neq 0$ states because of the centrifugal term $\sim r^{-2}[16-$ 17]. the Schrodinger equation can only be solved approximately for different suitable approximation scheme. One of the suitable approximation scheme is conventionally proposed by Greene and Aldrich.[18, 19]

In this paper we will attempt to solve the Schrodinger equation for a charged particle moving in a field governed by modified Poschl-Teller potential [20]with simultaneously presence of trigonometric Rosen-Morse potential[21]in term of finite Romanovski polynomial. The modified Poschl-Teller potential is hyperbolic potential. A class of hyperbolic potentials play the essential roles in interatomic and intermolecular forces [2223] and can be used to describe molecular vibrations. Some of these hyperbolic potential are exactly solvable or quasi - exactly solvable and their bound state solutions have been reported[24-25]. The trigonometric Rosen- 
Morse potential proposed by N. Rosen and P.M. Morse [21] in 1932. Trigonometric Rosen-Morse potential was used to describe the essential of the QCD quark-gluon dynamics in the regime suited of the asymptotical freedom of the quarks [26-28]. The approximate bound state solution for modified Poschl-Teller and trigonometric Rosen-Morse potentials have also been studied for $l$-state solution [19, 27,29].

This paper is organized as follows. In section 2, we review the finite Romanovski polynomials briefly. In section 3, we find the bound state energy solution and radial wave function from the solution of the radial Schrodinger equation, and the angular wave functions are derived in section 4 . A brief conclusion is presented in section 5 .

\section{Review of formulas for finite Romanovski Polynomials}

The one-dimensional Schrodinger equation of any shape invariant potential can be reduced into hypergeometric of confluent hypergeometric type differential equation by suitable variable transformation [1516]. The hypergeometric type differential equation, which can be solved using finite Romanovski polynomials that was developed by Romanovski $[12,14,15]$ is presented as

$\sigma \frac{\partial^{2} y_{n}}{\partial s^{2}}+\tau \frac{\partial y_{n}}{\partial s}+\lambda y_{n}=0$

where $\sigma(s)=a s^{2}+b s+c ; \quad \tau=d s+e$ and $\quad-\{n(n-1)+2 n(1-p)\}=\lambda=\lambda_{n}$

Equation (1) is described in the textbook by Nikiforov-Uvarov where it is cast into self adjoint form and its weight function,$w(s)$, satisfies the so called Pearson differential equation

$\frac{d(\sigma(s) w(s))}{d s}=\tau(s) w(s)$

The weight function is obtained by solving the Pearson differential equation, that is

$w(s)=\exp \left(\left[\int \frac{(d-2 a) s+(e-b)}{a s^{2}+b s+c} d s\right.\right.$

The corresponding polynomials are classified according to the weight function, and are built up from the Rodrigues representation as

$y_{n}=\frac{1}{w(s)} \frac{d^{n}}{d s^{n}}\left\{\left(a s^{2}+b s+c\right)^{n} w(s)\right\}$

For Romanovski polynomial, the values of parameters in equation (4) are:

$a=1, b=0, c=1, d=2(1-p)$ and $e=q$ with $p>0(6)$

By inserting equation (6) into equation (4) we obtain the weight function

$$
w(s)=\exp \left(\frac{(d-2 a) s+(c-b)}{a s^{2}+b s+c}=\exp \left(\frac{(2-2 p-2) s+q}{s^{2}+1} d s\right.\right.
$$

$w(s)=\left(1+s^{2}\right)^{-p} e^{q \tan ^{-1}(s)}$

This weight function first reported by Routh [15] and then by Romanovski. The polynomial associated with equation (7) are named after Romanovski and will be denoted by $R_{n}^{(p, q)}(s)$. Due to the decrease of the weight function by $s^{-2 p}$, integral of the type

$\int_{-\infty}^{\infty} w^{(p, q)} R_{m}^{(p, q)}(s) R_{m}^{(p, q)}(s) d s$

will be convergent only if $\quad m^{\prime}+m<2 p-1$

This means that only a finite number of Romanovski polynomials are orthogonal.

The differential equation satisfied by Romanovski Polynomial obtained by inserting equations (2) and (6) into equation (1) is

$\left(1+s^{2}\right) \frac{\partial^{2} R_{n}^{(p, q)}(s)}{\partial s^{2}}+\{2 s(-p+1)+q\} \frac{\partial R_{n}^{(p, q)}(s)}{\partial s}-\{n(n-1)+2 n(1-p)\} R_{n}^{(p, q)}(s)=0(10)$

where $y_{n}=R_{n}^{(p, q)}(s)$. The Schrodinger equation of the potential of interest will be reduced into the form which is similar to equation (10) by an appropriate transformation of variable, $r=f(s)$, and by introducing a new wave function which is given as

$\psi(r)=g_{n}(s)=\left(1+s^{2}\right)^{\frac{\beta}{2}} e^{\frac{-\alpha}{2} \tan ^{-1} s} D_{n}^{(\beta, \alpha)}(s)$

The eigen function in equation (11) is the solution of Schrodinger equation for potential interest where $D_{n}^{(\beta, \alpha)}(s)=R_{n}^{(p, q)}(s)$

The Romanovski polynomials obtained from Rodrigues formula with the weight function in equation (5) is expressed as

$R_{n}^{(p, q)}(s)=D_{n}^{(\beta, \alpha)}(s)=\frac{1}{\left(1+s^{2}\right)^{-p} e^{q t a n^{-1}(s)}} \frac{d^{n}}{d s^{n}}\left\{\left(1+s^{2}\right)^{n}\left(1+s^{2}\right)^{-p} e^{q \tan ^{-1}(s)}\right\}$

If the wave function of the nth level in equation (11) is rewritten as

$\psi_{n}(r)=\frac{1}{\sqrt{\frac{d f(s)}{d s}}}\left(1+s^{2}\right)^{\frac{-p}{2}} e^{\frac{q}{2} \tan ^{-1}(s)} R_{n}^{(p, q)}(s)$

then the orthogonality integral of the wave functions expressed in equation (14) gives rise to orthogonality integral of the finite Romanovski polynomials, that is 
$\int_{0}^{\infty} \psi_{n}(r) \psi_{n^{\prime}}(r) d r=\int_{-\infty}^{\infty} w^{(p, q)} R_{n}^{(p, q)}(s) R_{n^{\prime}}^{(p, q)}(s) d s$

In this case the values of $p$ and $q$ are not $\mathrm{n}$-dependence where $\mathrm{n}$ is the degree of polynomials. However, if equation (9) is not fulfilled then the Romanovskipolynomials is infinity [15].

\section{Solution of the Schrodinger Equation for Hyperbolic Poschl-Teller-Trigonometric Poschl-Teller Non-Central Potential using Romanovski Polynomials}

Modified Poschl-Teller potential plus trigonometric Rosen Morse non-central potential is the potential where both potentials present simultaneously in the quantum system. This non-central potential is expressed as

$V(r, \theta)=\frac{\hbar^{2}}{2 M \gamma^{2}}\left(\frac{\varkappa(\varkappa-1)}{\sinh ^{2} \frac{r}{\gamma}}-\frac{\lambda(\lambda+1)}{\cosh ^{2} \frac{r}{\gamma}}\right)+\frac{\hbar^{2}}{2 \mu r^{2}}\left(\frac{v(v+1)}{\sin ^{2} \theta}-2 \mu \cot \theta\right)$

where $\lambda>\varkappa-1 ; 0 \leq r \leq \infty, \quad v>1, \mu>1, \quad 0 \leq \theta \leq \frac{\pi}{2}$

The three dimensional Schrodinger equation for hyperbolic Poschl-Teller - trigonometric Rosen-Morse noncentral potential is written as

$-\frac{\hbar^{2}}{2 M}\left\{\frac{1}{r^{2}} \frac{\partial}{\partial r}\left(r^{2} \frac{\partial}{\partial r}\right)+\frac{1}{r^{2} \sin \theta} \frac{\partial}{\partial \theta}\left(\sin \theta \frac{\partial}{\partial \theta}\right)+\frac{1}{r^{2} \sin ^{2} \theta} \frac{\partial^{2}}{\partial \varphi^{2}}\right) \psi(r, \theta, \varphi)+\left\{\frac{\hbar^{2}}{2 M \gamma^{2}}\left(\frac{\varkappa(\varkappa-1)}{\sinh ^{2} \frac{r}{\gamma}}-\frac{\lambda(\lambda+1)}{\cosh ^{2} \frac{r}{\gamma}}\right)+\frac{\hbar^{2}}{2 M r^{2}}\left(\frac{v(v+1)}{\sin ^{2} \theta}-\right.\right.$

$2 \mu \cot \theta \psi r, \theta, \varphi=E \psi r, \theta, \varphi$

Equation (17) is solved using separation variable method since the non-central potential is separable. By setting $\psi(r, \theta, \varphi)=R(r) P(\theta) \Phi(\varphi)$ in equation (17) we obtain

$\frac{1}{R} \frac{\partial}{\partial r}\left(r^{2} \frac{\partial R}{\partial r}\right)-\frac{r^{2}}{\gamma^{2}}\left(\frac{\varkappa(\varkappa-1)}{\sinh ^{2} \frac{r}{\gamma}}-\frac{\lambda(\lambda+1)}{\cosh ^{2} \frac{r}{\gamma}}\right)+\frac{2 M}{\hbar^{2}} E r^{2}=-\frac{1}{P \sin \theta} \frac{\partial}{\partial \theta}\left(\sin \theta \frac{\partial P}{\partial \theta}\right)-\frac{1}{\sin ^{2} \theta} \frac{1}{\Phi} \frac{\partial^{2} \Phi}{\partial \varphi^{2}}+\left(\frac{v(v+1)}{\sin ^{2} \theta}-2 \mu \cot \theta\right)=$

$l(l+1)$

From equation (18) we obtain radial and angular Schrodinger equations as

$\frac{1}{r^{2}} \frac{\partial}{\partial r}\left(r^{2} \frac{\partial R}{\partial r}\right)-\frac{1}{\gamma^{2}}\left(\frac{\varkappa(\varkappa-1)}{\sinh ^{2} \frac{r}{\gamma}}-\frac{\lambda(\lambda+1)}{\cosh ^{2} \frac{r}{\gamma}}\right) R+\frac{2 M}{\hbar^{2}} E R-\frac{l(l+1)}{r^{2}} R=0$

and

$\frac{1}{\sin \theta} \frac{\partial}{\partial \theta}\left(\sin \theta \frac{\partial P}{\partial \theta}\right)-\frac{m^{2} P}{\sin ^{2} \theta}-\left(\frac{v(v+1)}{\sin ^{2} \theta}-2 \mu \cot \theta\right) P+l(l+1) P=0$

where we have set $\frac{1}{\Phi} \frac{\partial^{2} \Phi}{\partial \varphi^{2}}=-m^{2}$ that gives azimuthal wave function as

$\Phi=\frac{1}{\sqrt{2 \pi}} e^{i m \varphi}, m=0, \pm 1, \pm 2, \pm 3, \ldots$.

\subsection{The Solution of Angular Schrodinger Equation}

The polar part of the Schrodinger equation expressed in equation (20) is rewritten as

$\frac{\partial^{2} P(\theta)}{\partial \theta^{2}}+\cot \theta \frac{\partial P(\theta)}{\partial \theta}-\left(\frac{v(v+1)+m^{2}}{\sin ^{2} \theta}-2 \mu \cot \theta\right) P(\theta)+l(l+1) P(\theta)=0$

To solve equation (22) we introduce a new variable $\cot \theta=s$ and equation (22) change into

$\left(\left(1+s^{2}\right) \frac{\partial^{2} P}{\partial s^{2}}+s \frac{\partial P}{\partial s}-\left\{\left(v(v+1)+m^{2}\right)-\frac{2 \mu x}{\left(1+s^{2}\right)}-\frac{l(l+1)}{\left(1+s^{2}\right)}\right\} P=0\right.$

Equation (23) is solved in terms of Romanovski polynomialby setting

$P(\theta)=g_{n}(s)=\left(1+s^{2}\right)^{\frac{\beta}{2}} e^{\frac{-\alpha}{2} \tan ^{-1} s} D_{n}^{(\beta, \alpha)}(s)$,

By inserting equation (24) into equation (23) we obtain

$$
0<s<\infty
$$

$\left(1+s^{2}\right) \frac{\partial^{2} D}{\partial s^{2}}+\{s(2 \beta+1)-\alpha\} \frac{\partial D}{\partial s}-\left\{\frac{\beta s \alpha-\frac{\alpha^{2}}{4}+\beta^{2}-\frac{\alpha s}{2}-\beta-2 \mu s-l(l+1)}{1+s^{2}}+v(v+1)+m^{2}-\beta^{2}-\beta+\beta\right\} D=0$

Equation (25) reduces to differential equation satisfied by Romanovsk
$\left(1+s^{2}\right) \frac{\partial^{2} D}{\partial s^{2}}+\{s(2 \beta+1)-\alpha\} \frac{\partial D}{\partial s}-\left\{v(v+1)+m^{2}-\beta^{2}\right\} D=0$

for $\beta s \alpha-\frac{\alpha^{2}}{4}+\beta^{2}-\frac{\alpha s}{2}-\beta-2 \mu s-l(l+1)=0$

By comparing equations (10) and (26) we obtain

$(2 \beta+1)=2(-p+1) ; \alpha=-q$ and $v(v+1)+m^{2}-\beta^{2}=n(n-1)+2 n(1-p)$

From equation (27) we have

$-\frac{\alpha^{2}}{4}+\beta^{2}-\beta-l(l+1)=0 ; \quad \beta \alpha-\frac{\alpha}{2}-2 \mu=0$ 
that give $\alpha^{2}=-2\left(l+\frac{1}{2}\right)^{2} \pm 2 \sqrt{\left(l+\frac{1}{2}\right)^{4}+4 \mu^{2}}$

$\operatorname{and}\left(\beta-\frac{1}{2}\right)^{2}=\frac{2 \mu^{2}}{-\left(l+\frac{1}{2}\right)^{2} \pm \sqrt{\left(l+\frac{1}{2}\right)^{4}+4 \mu^{2}}}$

Using equation (28) we obtain

$p=-\beta+\frac{1}{2} ; q=-\alpha$

and $v(v+1)+m^{2}=(\beta+n)^{2}$

Then from equation (33) we get

$\beta-\frac{1}{2}=\sqrt{v(v+1)+m^{2}}-n-\frac{1}{2}$ (i)

$\left(\beta-\frac{1}{2}\right)=-\sqrt{\nu(v+1)+m^{2}}-n-\frac{1}{2}$

By using equations (29) with (34) or with (35) we obtain

$\alpha_{n}=\frac{2 \mu}{\sqrt{v(v+1)+m^{2}}-n-\frac{1}{2}}$

or $\alpha_{n}=-\frac{2 \mu}{\sqrt{v(v+1)+m^{2}}+n+\frac{1}{2}}$

From equations (34), (35), and (31) we obtain

$$
\begin{aligned}
& \left(\sqrt{v(v+1)+m^{2}}-n-\frac{1}{2}\right)^{2}=\frac{2 \mu^{2}}{-\left(l+\frac{1}{2}\right)^{2} \pm \sqrt{\left(l+\frac{1}{2}\right)^{4}+4 \mu^{2}}} \\
& \operatorname{or}\left(-\sqrt{v(v+1)+m^{2}}-n-\frac{1}{2}\right)^{2}=\frac{2 \mu^{2}}{-\left(l+\frac{1}{2}\right)^{2} \pm \sqrt{\left(l+\frac{1}{2}\right)^{4}+4 \mu^{2}}}
\end{aligned}
$$

To have more physical meaning, the value of $\beta$ and $\alpha$ that satisfies the system are expressed in equation (35) and (37) therefore the value of $l$ that satisfies the system is obtained from equation (39) given as

$l+\frac{1}{2}=\sqrt{\left(\sqrt{v(v+1)+m^{2}}+n+\frac{1}{2}\right)^{2}-\frac{\mu^{2}}{\left(\sqrt{v(v+1)+m^{2}}+n+\frac{1}{2}\right)^{2}}}$

The weight function obtained from equations (7), (32), (35) and (37) is given as

$w^{\left(-\beta+\frac{1}{2},-\alpha\right)}=\left(1+s^{2}\right)^{\beta_{n}-\frac{1}{2}} e^{-\alpha_{n} \tan ^{-1} s}$

Using equations (13) and (41) we obtain the Romanovski polynomials given as

$$
\begin{gathered}
R_{n}^{(p, q)}(s)=D_{n}^{(\beta, \alpha)}(s)=\frac{1}{w^{\left(-\beta+\frac{1}{2},-\alpha\right)}} \frac{d^{n}}{d s^{n}}\left\{\left(1+s^{2}\right)^{n} w^{\left(-\beta+\frac{1}{2},-\alpha\right)}(s)\right\} \\
\operatorname{or}_{n}^{\left(-\beta+\frac{1}{2},-\alpha\right)}(s)=\frac{1}{\left(1+s^{2}\right)^{\beta_{n}-\frac{1}{2} e^{-\alpha_{n} \tan ^{-1} s}} \frac{d^{n}}{d s^{n}}\left\{\left(1+s^{2}\right)^{\beta_{n}-\frac{1}{2}+n} e^{-\alpha_{n} \tan ^{-1} s}\right\}}
\end{gathered}
$$

The angular wave function obtained from equations (11) and (42) is given as

$P_{n}\left(\theta=\cot ^{-1} s\right)=g_{n}(s)=\left(1+s^{2}\right)^{\frac{\beta_{n}}{2}} e^{\frac{-\alpha_{n}}{2} \tan ^{-1} s} R_{n}^{\left(-\beta+\frac{1}{2},-\alpha\right)}(s)$

The orthogonality integral of Schrodinger wave function can not give rise to the orthogonality integral of the Romanovski polynomial since the weight function is a function of the degree of the polynomial, $\mathrm{n}$.

$\int P_{n}(\theta) P_{n^{\prime}}(\theta) \sin \theta d \theta=\int\left(1+s^{2}\right)^{\frac{\beta_{n^{\prime}+\beta_{n}}}{2}-\frac{1}{2}} e^{-\frac{\left(\alpha_{n}{ }^{\left.\prime+\alpha_{n}\right)}\right.}{2} \tan ^{-1}(s)} R_{n}^{\left(-\beta+\frac{1}{2},-\alpha\right)}(s) R_{n^{\prime}}^{\left(-\beta+\frac{1}{2^{\prime}}, \alpha\right)}(s) \frac{d s}{1+s^{2}} \neq \int(1+$ s2) $\beta n-12 e-\alpha n t a n-1(s) R n-\beta+12,-\alpha s R n^{\prime}-\beta+12,-\alpha s d s$

Equation (41) shows that the orthogonality of Romanovski polynomials can not be produced from the orthogonality of wave function and so the Romnovski polynomials are infinite.

Construction of Romanovski polynomial

The first four Romanovski polynomial are constructed using equation (39)

$R_{0}^{\left(-\beta+\frac{1}{2},-\alpha\right)}(s)=1$

$R_{1}^{\left(-\beta+\frac{1}{2},-\alpha\right)}(s)=2\left(\beta_{1}+\frac{1}{2}\right) s-\alpha_{1}$

$R_{2}^{\left(-\beta+\frac{1}{2},-\alpha\right)}(s)=\left\{4\left(\beta_{2}+1 \frac{1}{2}\right)\left(\beta_{2}+1\right) s^{2}-4 \alpha_{2}\left(\beta_{2}+1\right) s+\alpha_{2}^{2}+2\left(\beta_{2}+1 \frac{1}{2}\right)\right\}$

$R_{3}^{\left(-\beta_{3}+\frac{1}{2},-\alpha_{3}\right)}(s)=8\left(\beta_{3}+2 \frac{1}{2}\right)\left(\beta_{3}+2\right)\left(\beta_{3}+1 \frac{1}{2}\right) s^{3}-12 \alpha_{3}\left(\beta_{3}+1 \frac{1}{2}\right)\left(\beta_{3}+2\right) s^{2}+6\left\{\alpha_{3}^{2}+\right.$ 
The Romanovski polynomials expressed in equations (45-48) can be constructed manually or using computer programming with mathematica software. If Rosen-Morse noncentral potential is absent then

$\beta_{n}=-m-n ; \alpha_{n}=0 l=\sqrt{\left(m+n_{l}+\frac{1}{2}\right)^{2}}-\frac{1}{2}=n_{l}+m$

and the angular wave functions reduce to associated Legendre polynomials, as shown in $1^{\text {st }}, 5^{\text {th }}, 6^{\text {th }}$ rows in Table 1. The graphs of the angular wave function listed in the first four rows are shown in Figure 1.

Table1. The Romanovski polynomials and its corresponding angular wave functions for modified PoschlTeller plus Rosen-Morse non-central potential

\begin{tabular}{|c|c|c|c|c|c|c|c|}
\hline No & $\mathrm{n}_{1}$ & $\mathrm{~m}$ & $v$ & $\mu$ & 1 & $R_{n}^{\left(-\beta+\frac{1}{2},-\alpha\right)}(s)$ & $\begin{array}{lll}\text { Non-central } & \text { Associated } & \text { Legendre } \\
\text { Polynomials } & & \\
\end{array}$ \\
\hline 1 & 1 & 1 & 0 & 0 & 2 & $-3 \cot \theta$ & $-3 \sin \theta \cos \theta$ \\
\hline 2 & 1 & 1 & 2 & 0 & 3.65 & $-6.3 \cot \theta$ & $-3(\sin \theta)^{2.65} \cos \theta$ \\
\hline 3 & 1 & 1 & 0 & 2 & 1.4 & $-3 \cot \theta+1.6$ & $(-3 \cot \theta+1.6)(\sin \theta)^{2} e^{o .8 \tan ^{-1}(\cot \theta)}$ \\
\hline 4 & 1 & 1 & 2 & 2 & 3.62 & $-6.3 \cot \theta+0.96$ & $\begin{array}{l}(-6.3 \cos \theta \\
+0.96 \sin \theta)(\sin \theta)^{2.65} e^{o .48 \tan ^{-1}(\cot \theta)}\end{array}$ \\
\hline 5 & 1 & 2 & 0 & 0 & 3 & $-5 \cot \theta$ & $-5(\sin \theta)^{2} \cos \theta$ \\
\hline 6 & 2 & 1 & 0 & 0 & 3 & $12 \cot ^{2} \theta-3$ & $3 \sin \theta\left(4 \cos ^{2} \theta-\sin ^{2} \theta\right)$ \\
\hline
\end{tabular}

The angular wave functions with/without the presence of trigonometric Rosen-Morse non-central potential are listed in Table 1. The first four of the angular momentum at the first four rows are graphed usingMathematica software as shown in Figure 1. The effect of the $\csc ^{2} \theta$ term alone which assigned by the value of $\beta$ to the associated Legendre function causes the change in direction and increase of the angular momentum vector in the interval $0<\theta<\pi$. On the other hand the effect of $\cot \theta$ term alone causes the decrease of the angular momentum vector in the interval $0<\theta<\frac{\pi}{2}$ but increases the angular momentum vector in the interval $\frac{\pi}{2}<\theta<$ $\pi$. This term is considered to be the perturbed factor to the original wave (spherical harmonics wave function). Figure 1shows that the shape of the angular wave function graphs do not change by the presence of the RosenMorse potential but change the length of the angular momentum vector. [27]
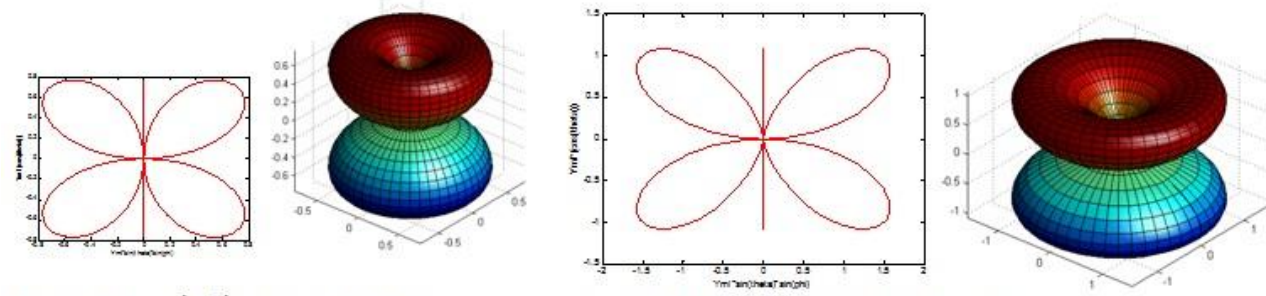

Figure (1a) $\quad\left|Y_{2}^{1}\right|=3 \cos \theta \sin \theta$

Figure(1b) $\left|Y_{3.65}^{1}\right|=3(\sin \theta)^{2.65} \cos \theta$

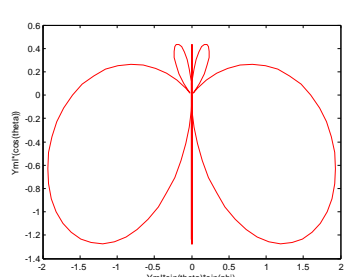

Figure(1c)

$\left|\boldsymbol{Y}_{\mathbf{1 . 4}}^{\mathbf{1}}\right|=(-3 \cos \theta+1.6 \sin \theta) \sin \theta e^{o .8 \tan ^{-1}(\cot \theta)}$
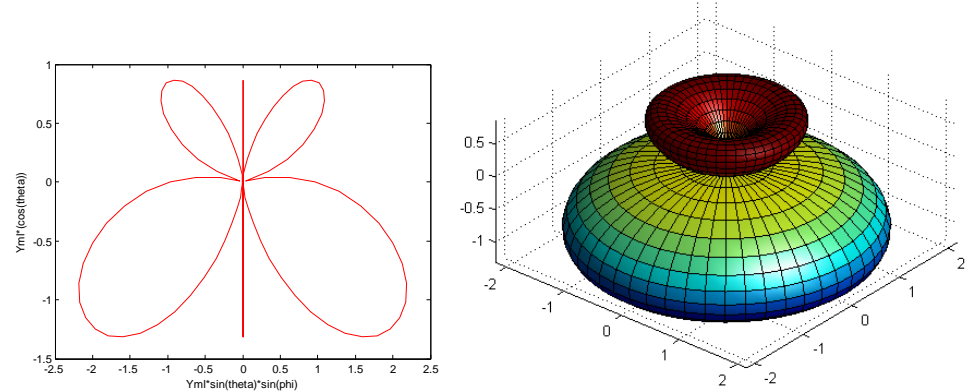

Figure 1 Thepolar diagram of the orbital angular momentum eigen function $Y_{l m}$ and three dimensional polar representations of the absolute value of the angular function. (1a) The absent of Rosen-Morse non-central potential, the effect of $\csc ^{2} \theta$ term causes the length of the angular momentum vector increases in $(1 \mathrm{~b})$, the $\cot \theta$ term decreases the length of angular momentum vector of $0<\theta<\frac{\pi}{2}$ but increases the length at $\frac{\pi}{2}<\theta<\pi$, and (1d) shows the total effect of both terms that decreases the length of angular momentum vector of $0<\theta<\frac{\pi}{2}$. increases the length at $\frac{\pi}{2}<\theta<\pi$.

Figure (1d)

$\left|Y_{3,62}^{1}\right|=(6.3 \cos \theta+0.96 \sin \theta)(\sin \theta)^{2.65} e^{0.48 \tan ^{-1}(\cot \theta)}$ Www.iosrjournals.org 


\subsection{Solution of radial Schrodinger equation}

To solve theradial Schrodinger equation in equation (19) we use the approximation value for centrifugal term ${ }^{[14-}$ 15],$\frac{1}{r^{2}} \cong \frac{1}{\gamma^{2}}\left(d_{0}+\frac{1}{\sinh ^{2} \frac{r}{\gamma}}\right)$ for $\frac{r}{\alpha}<<1$, with $d_{0}=\frac{1}{12}$. By setting $\mathrm{R}(r)=\frac{\chi(r)}{r}, \epsilon^{2}=-\frac{2 m}{\hbar^{2}} E$ and by using the approximation value for centrifugal term, equation (19) becomes

$\frac{d^{2} \chi(r)}{d r^{2}}-\left\{\frac{\varkappa(\varkappa-1)+l(l+1)}{\gamma^{2} \sinh ^{2} \frac{r}{\gamma}}-\frac{\lambda(\lambda+1)}{\alpha^{2} \cosh ^{2} \frac{r}{\gamma}}+\epsilon^{2}+\frac{l(l+1) d_{0}}{\gamma^{2}}\right\} \chi(r)=0$

By making a change of variable in equation (49), $\cosh \frac{2 r}{\gamma}=i x$, we have

$\left(1+x^{2}\right) \frac{\partial^{2} \chi}{\partial x^{2}}+x \frac{\partial \chi}{\partial x}+\left\{\frac{\varkappa(\varkappa-1)+l(l+1)+\lambda(\lambda+1)}{2\left(1+x^{2}\right)}+\frac{\varkappa(\varkappa-1)+l(l+1)-\lambda(\lambda+1)}{2\left(1+x^{2}\right)} i x-\frac{\gamma^{2} \epsilon^{2}+l(l+1) d_{0}}{4}\right\} \chi=0(50)$

To solve equation (50) in terms of Romanovski Polynomials, according to equation (11) we set the eigenfunction of the radial part as

$\chi(r)=g_{n}(x)=\left(1+x^{2}\right)^{\frac{\beta}{2}} e^{\frac{-\alpha}{2} \tan ^{-1} x} D_{n}^{(\beta, \alpha)}(x)$,

with1 $<i x<\infty$

By insertying equation (51) into equation (50) we have

$\left(1+x^{2}\right) \frac{\partial^{2} D}{\partial x^{2}}+\{x(2 \beta+1)-\alpha\} \frac{\partial D}{\partial x}-\left\{\frac{\left.\mathbf{2} \boldsymbol{\beta} \boldsymbol{x} \boldsymbol{\alpha}-\boldsymbol{x} \boldsymbol{\alpha}-\frac{\alpha^{2}}{2}+2 \beta^{2}-\mathbf{2} \boldsymbol{\beta}-\{(\varkappa(\varkappa-1)+l(l+1)-\lambda(\lambda+1)\}) i x-\{\varkappa(\varkappa-1)+l(l+1)+\lambda(\lambda+1))\right\}}{2\left(1+x^{2}\right)}+\right.$

$\gamma 2 \epsilon 2+l l+1 d 04-\beta 2 D=0$

By comparing equation (52) and equation (10) we obtain

$-\left\{\left(\mathcal{H}-\frac{1}{2}\right)^{2}+l(l+1)+\left(\lambda+\frac{1}{2}\right)^{2}\right\}-\frac{\alpha^{2}}{2}+2\left(\beta-\frac{1}{2}\right)^{2}=0$

$-\left(\left(\varkappa-\frac{1}{2}\right)^{2}+l(l+1)-\left(\lambda+\frac{1}{2}\right)^{2}\right) i+2 \beta \alpha-\alpha=0$

$(2 \beta+1)=2(-p+1) \alpha=-q$

$\frac{\gamma^{2} \epsilon^{2}+l(l+1) d_{0}}{4}-\beta^{2}=n(n-1)+2 n\left(\beta+\frac{1}{2}\right)$

and equation (52) becomes

To simplify the solution of equations (53)-(56) we set

$\left(\varkappa-\frac{1}{2}\right)^{2}+l(l+1)=A,\left(\lambda+\frac{1}{2}\right)^{2}=B$

and from equations (53) and (54) we obtain

$\alpha= \pm i(\sqrt{A} \mp \sqrt{B})$ and $\left(\beta-\frac{1}{2}\right)=\frac{(A-B)}{2 \alpha} i=\frac{(A-B)}{ \pm 2 i(\sqrt{A} \mp \sqrt{B})}$

To have more physical meaning the proper choice of the values of $\alpha$ and $\beta$ from equation (58) are

$\beta=\frac{\sqrt{A}-\sqrt{B}+1}{2}=\frac{\sqrt{\left(\varkappa-\frac{1}{2}\right)^{2}+l(l+1)}-\lambda+\frac{1}{2}}{2}$

and $\alpha=i(\sqrt{A}+\sqrt{B})=i\left(\sqrt{\left(\varkappa-\frac{1}{2}\right)^{2}+l(l+1)}+\lambda+\frac{1}{2}\right)$

From equation (55) we obtain

$\beta=\frac{1}{2}-p o r p=-\beta+\frac{1}{2}$ and $\alpha=-q$

and from equation (56) and (61) we have

$\frac{-2 M E}{\hbar^{2}} \frac{\gamma^{2}}{4}=(\beta+n)^{2}-\frac{l(l+1) d_{0}}{4}$

Thus the energy spectrum of the system obtained from equations (59) and (62) is

$E_{n}=-\frac{\hbar^{2}}{2 M \gamma^{2}}\left\{\left(\lambda-\sqrt{\left(\varkappa-\frac{1}{2}\right)^{2}+l(l+1)}-\frac{1}{2}-2 n\right)^{2}-l(l+1) d_{0}\right\}$

The energy spectrum in equation (63) reduces to the energy of modified Poschl-Teller potential for $l=0$ which is given as

$E_{n}=-\frac{\hbar^{2}}{2 M \gamma^{2}}\left\{(\lambda-\varkappa-2 n)^{2}\right\}$

To determine the radial wave function of the system, firstly we find the weight function from equations (7), (59), (60) and (61) as

$w^{(p, q)}=w^{\left(-\beta+\frac{1}{2},-\alpha\right)}=\left(1+x^{2}\right)^{\frac{(\sqrt{A}-\sqrt{B})}{2}} e^{-i(\sqrt{A}+\sqrt{B}) \tan ^{-1} x}$

and its wave function expressed in Romanovski polynomials obtained from equations (13) and (65) is 
$R_{n}^{\left(-\beta+\frac{1}{2},-\alpha\right)}(x)=\frac{1}{\left(1+x^{2}\right)^{\frac{(\sqrt{A}-\sqrt{B})}{2}} e^{-i(\sqrt{A}+\sqrt{B}) \tan ^{-1} x}} \frac{d^{n}}{d x^{n}}\left\{\left(\left(1+x^{2}\right)^{\frac{(\sqrt{A}-\sqrt{B})}{2}+n} e^{-i(\sqrt{A}+\sqrt{B}) \tan ^{-1} x}\right\}\right.$

By inserting equations (59), (60) and (61) into equation (51) we obtain the eigenfunction as

$\chi(r)=g_{n}(x)=\left(1+x^{2}\right)^{\frac{(\sqrt{A}-\sqrt{B})+1}{4}} e^{-\frac{i}{2}(\sqrt{A}+\sqrt{B}) \tan ^{-1} x} R_{n}^{\left(-\beta+\frac{1}{2},-\alpha\right)}(x)$

To observe the orthogonality integral of Romanovski polynomials, equation (67) is rewritten

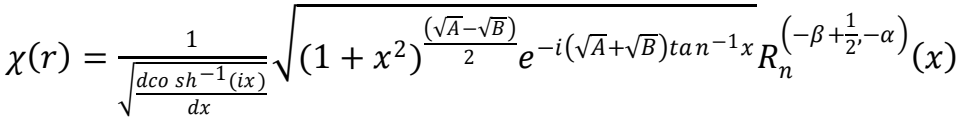

where $\frac{d r}{d x}=\gamma \frac{d \cos h^{-1}(i x)}{d x}=\frac{\gamma}{\sqrt{1+x^{2}}}$

The orthogonality integral of radial wave functions that will produce the orthogonality integral of Romanovski polynomials given as

$$
\begin{array}{r}
\int \chi_{n}(r) \chi_{n}{ }^{\prime}(r) d r=\int_{0}^{\infty} R_{n}(r) R_{n^{\prime}}(r) r^{2} d r \\
=\gamma \int_{1}^{\infty}\left(1+x^{2}\right)^{\frac{(\sqrt{A}-\sqrt{B})}{2}} e^{-i(\sqrt{A}+\sqrt{B}) \tan ^{-1} x} R_{n}^{\left(-\beta+\frac{1}{2},-\alpha\right)}(x) R_{n^{\prime}}^{\left(-\beta+\frac{1}{2^{\prime}},-\alpha\right)} d x
\end{array}
$$

The integral in equation (69) will be convergent only if $n+n^{\prime}<-(\sqrt{A}-\sqrt{B}+1)$

or2 $n<\lambda-\sqrt{\left(\varkappa-\frac{1}{2}\right)^{2}+l(l+1)}-\frac{1}{2}$

where $n$ is the degree of the polynomials which is non-negative integer. Equation (70) shows the condition that the number of Romanovski polynomials that are orthogonal is finite.

Construction of Romanovski Polynomial

The first four Romanovski Polynomials for radial wave functions obtained using equation (66) are

$R_{0}^{\left(-\beta+\frac{1}{2},-\alpha\right)}(x)=1$

$R_{1}^{\left(-\beta+\frac{1}{2},-\alpha\right)}(x)=(\sqrt{A}-\sqrt{B}+2) x-i(\sqrt{A}+\sqrt{B})$

$R_{2}^{\left(-\beta+\frac{1}{2},-\alpha\right)}(x)=\left[(\sqrt{A}-\sqrt{B}+4)(\sqrt{A}-\sqrt{B}+3) x^{2}-2 i x(\sqrt{A}+\sqrt{B})(\sqrt{A}-\sqrt{B}+3)-(\sqrt{A}+\sqrt{B})^{2}+\right.$

Table 2.Romanovski Polynomial and the corresponding radial wave function for $n_{l}=1,2$ and $m=1,2$

\begin{tabular}{|c|c|c|c|c|c|c|c|c|c|c|}
\hline N0 & $n_{l}$ & $m$ & $v$ & $\mu$ & $l$ & $\lambda$ & $\varkappa$ & $n_{r}$ & $\begin{array}{l}\text { Romanovski } \\
\text { Polynomials } \\
R_{n}^{\left(-\beta+\frac{1}{2},-\alpha\right)}(x)\end{array}$ & Radial wave function, $\chi(r)$ \\
\hline \multirow[t]{3}{*}{1} & \multirow[t]{3}{*}{1} & \multirow[t]{3}{*}{1} & \multirow[t]{3}{*}{0} & \multirow[t]{3}{*}{0} & \multirow[t]{3}{*}{2} & \multirow[t]{3}{*}{8} & \multirow[t]{3}{*}{2} & 0 & 1 & $i^{3.36} 2^{-2.32} \sinh ^{3.36} \frac{r}{\gamma} \cosh ^{-8} \frac{r}{\gamma}$ \\
\hline & & & & & & & & 1 & $-3.63 x-11.37 i$ & $\begin{array}{l}i^{4.36} 2^{-2.32} \sinh ^{3.36} \frac{r}{\gamma} \cosh ^{-8} \frac{r}{\gamma}(3.63 \cos \\
-11.37)\end{array}$ \\
\hline & & & & & & & & 2 & $\begin{array}{l}4.29 x^{2} \\
+59.8 i x \\
-130.9\end{array}$ & $\begin{array}{l}i^{3.36} 2^{-2.32} \sinh ^{3.36} \frac{r}{\gamma} \cosh ^{-8} \frac{r}{\gamma}(-4.29 c \\
\left.+59.8 \cosh \frac{2 r}{\gamma}-130.9\right)\end{array}$ \\
\hline \multirow[t]{2}{*}{2} & \multirow[t]{2}{*}{1} & \multirow[t]{2}{*}{1} & \multirow[t]{2}{*}{2} & \multirow[t]{2}{*}{0} & \multirow[t]{2}{*}{3.65} & \multirow[t]{2}{*}{8} & \multirow[t]{2}{*}{2} & 0 & 1 & $i^{4.88} 2^{-1.56} \sinh ^{4.88} \frac{r}{\gamma} \cosh ^{-8} \frac{r}{\gamma}$ \\
\hline & & & & & & & & 1 & $\begin{array}{l}-2.12 x \\
-12.88 i\end{array}$ & $\begin{array}{l}i^{5.88} 2^{-1.56} \sinh ^{4.88} \frac{r}{\gamma} \cosh ^{-8} \frac{r}{\gamma}(2.12 \cos \\
-12.88)\end{array}$ \\
\hline 3 & 1 & 1 & 0 & 2 & 1.4 & 8 & 2 & 0 & 1 & $i^{2.88} 2^{-1.56} \sinh ^{2.88} \frac{r}{\gamma} \cosh ^{-8} \frac{r}{\gamma}$ \\
\hline
\end{tabular}
$\boldsymbol{v}, \boldsymbol{\mu}=0,2,2, \lambda=8 ; \varkappa=2$ 


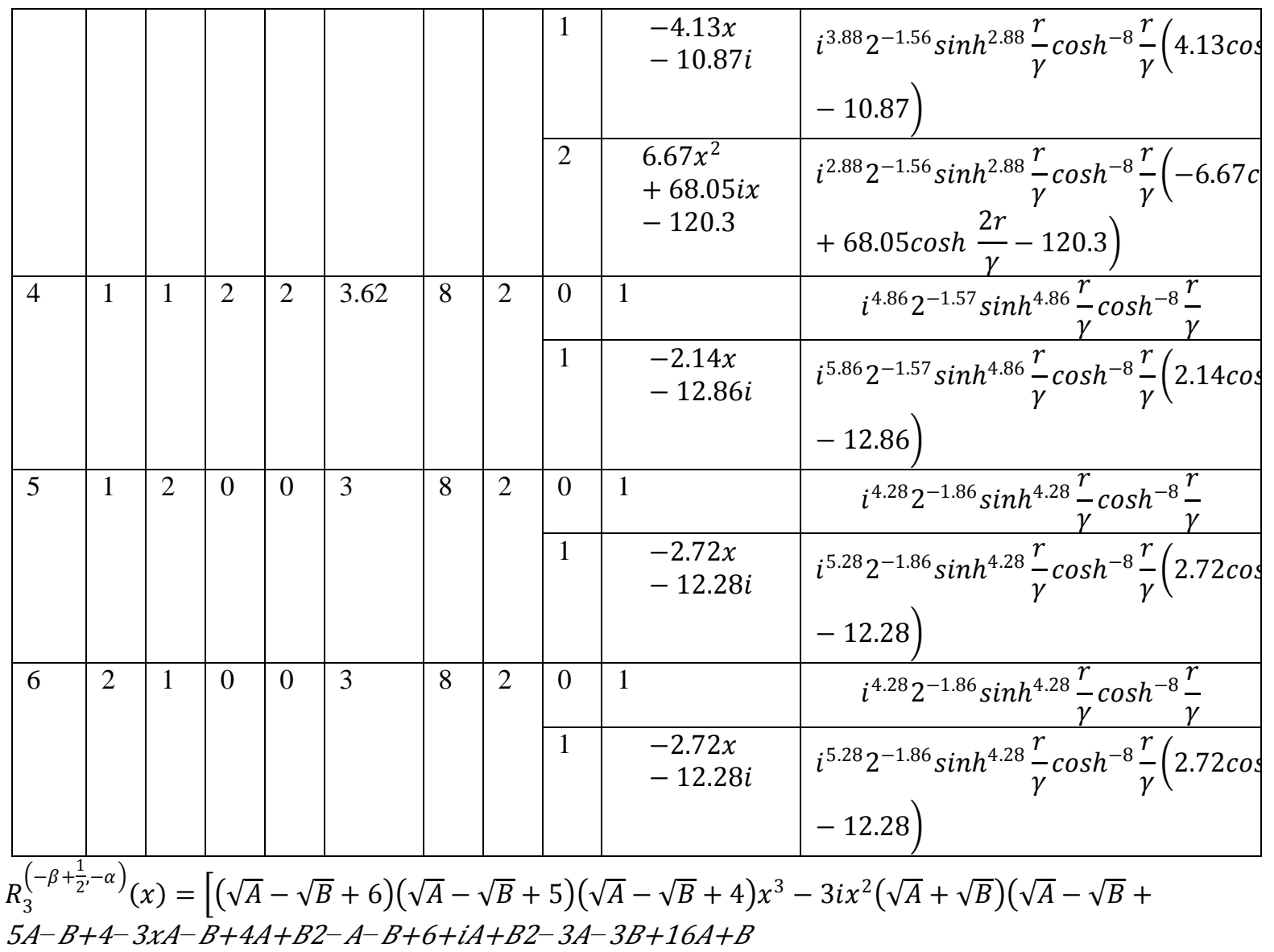

By setting $l=0$ in equation (67) we obtain the wave function of modified Poschl-Teller potential, equation (70) becomes $2 n_{r}<-\mathcal{H}+\lambda$, and for $n_{r}=0$ we obtain the ground state wave function for modified Poschl-Teller potential given as

$$
\begin{aligned}
& \chi_{00}(r)=\left(1-\cosh ^{2} \frac{2 r}{\gamma}\right)^{\frac{\varkappa-\frac{1}{2}-\lambda+\frac{1}{2}}{4}} e^{-i \frac{(\varkappa+\lambda)}{2} \tan ^{-1} x}=C\left(\sinh \frac{r}{\gamma}\right)^{\varkappa}\left(\cosh \frac{r}{\gamma}\right)^{-\lambda} \\
& \text { wheretan }^{-1} x=-\operatorname{itanh}^{-1}\left(\cosh \frac{2 r}{\gamma}\right)=\frac{-i}{2} \ln \left(\frac{1+\cosh \frac{2 r}{\gamma}}{1-\cosh \frac{2 r}{\gamma}}\right)
\end{aligned}
$$

The lowest wave function or the ground state wave function expressed in equation (75) is in agreement with the result determined using SUSY QM and NU method.

For $l$-wave, the values of $l$ depend on the value of the trigonometric Rosen-Morse potential's parameters as shown in equations (36) and (58). The $l$-waves are obtained by using equations (36), (58), (66), (67), and applying the condition in equation (70) for finite Romanovski polynomial. The number of Romanovski polynomials for radial wave function that are orthogonal depend on the values of the parameters of both potentials and the degree of Romanovski polynomial for angular wave function and the original quantum magnetic number, $\mathrm{m}$. Table 2 shows the Romanovski polynomials for radial wave function with and without the presence of Rosen-Morse non-central potential that are orthogonal.

The values of $n_{r}=0,1$, and 2obtained by inserting the values of the parameters, $\lambda$, and $\mathcal{\varkappa}$, that are listed in the first row of Table 2 into equation (70), for $\lambda=8$ and $\varkappa=2$, therefore we get three orthogonal radial wave function corresponding to three values of $n_{r}$ as shown at the first three sub-rows at the last right column, and the values of parameters $l=3,65, \lambda=8$ and $\varkappa=2$ as listed at the second row give the values of $n_{r}=0,1$. These two examples show that any set of parameters determine the number of Romanovski polynomials that are orthogonal, therefore there is limit number of the orthogonal wave function or the number of orthogonal wave function is finite.

\section{Conclusion}

The Schrodinger equation for modified Poschl-Teller potential with simultaneously the presence of Rosen-Morse non-central potential is solved approximately using Romanovski polynomial. The energy 
spectrum of non-central system is expressed in the closed form and is reduced to the energy spectrum for modified Poschl-Teller potential for $l=0$. The angular wave functions are expressed in terms of Romanovski polynomials, and reduced to associated Legendre polynomials by the absent of trigonometric Poschl-Teller noncentral potential. The radial wave functions are expressed in term of finite Romanovski polynomials and reduced to the wave function of modified Poschl-Teller potential for $l=0$. The presence of $\cot \theta$ decreases the length of angular momentum vector but the $\operatorname{cosec}^{2} \theta$ term causes the increase of the angular momentum vector length.

\section{Acknowledgement}

This work is partially supported by HibahPascasarjanaSebelasMaret University grant No : 2340/UN 27.10/PG/2012.

\section{References}

[1] V.M. Villalba, Elsevier Science, Bound State of The Hydrogen Atom in The Presence of A Magnetic Monopole Field and An Ahoronov-Bohm Potential, Phys. Lett. A (193), 1994, 218-222.

[2] Y. Aharonov and D. Bohm, Significance of electromagnetic potentials in quantum theory, Phys. Rev. (115), $1959,485$.

[3] L. V. Hoang, L.X. Hai, L.I. Komarov and T. S. Romanova, Relativistic Analogy of the Aharonov-BohmEffect in the Presence of Coulomb Field and Magnetic Charge, J. Phys. a (25), 1992, 6461.

[4] M. Movahedi and M. Hamzavi, Relativistic Scattering State Solutions of the Makarov Potential, Int. J. Phys. Sci.6 (4), $2011,89$.

[5] B. Gonul and I. Zorba, Supersymmetric Solutions of Non-Central Potentials, Phys. Lett. A 269 ( 2000$) 83$.

[6] S. Ferrer, A unified treatment for some ring-shaped potentials as a generalized 4-D isotropic oscillator, Mono. Acad. Ci. Zar, (30), 2006, 11-21.

[7] C.Y Chen and S. H. Dong,Exactly complete solutions of the Coulomb potential plus a new ring-shaped potential, Phys. Lett. A (335), 2005, 374

[8] H.X. Quan, L. Guang, W.Z. Min, N.L. Bin, and M. Yan, Solving Dirac Equation with New Ring-Shaped Non-Spherical Harmonic Oscillator Potential, Com. Theor. Phys. (China) (53) ,2010, 242-246.

[9] J.C.Sheng, Z.Ying, Z.X. Lin, S.L.Tian, Identity for the Exponential-Type Molecule Potentials and the Supersymmetry Shape Invariance, Com. Theor. Phys. (36), 2001, 641-646

[10] J. Sadeghi, Factorization Method and Solution of the Non-Central ModifedKratzer Potential, Act. Phys. Polonica, A 112 (1), 2007, 23.

[11] J. Sadeghi and B. Pourhassan, Exact solution of the non-central modified Kratzer potential plus a ring-shaped like potential by the factorization method, EJTP, 5 (193), 2008.

[12] A. F. Nikiforov and U. B. Uvarov, Special Function in Mathematical Physics (Birkhausa, Basel 1988).

[13] W. Koepf and M. Masjed-Jamei, Integral Transform and Special Functions, 17, 2006, 559.

[14] A. P. Raposo, H. J. Weber, D. E. Alvarez-Castillo, and M. Kirchbach,Romanovski Polynomials in Selected Physics Problems, arXiv:0706.3897v1 [quant-ph], 2007.

[15] D. Agboola, Schrödinger Equation with Hulth'en Potential Plus Ring-Shaped Potential, Commun. Theor. Phys. 55, 2011, 972-976.

[16] S.M. Ikhdair, Quantization Rule Solution to The Hulthen Potential in Arbitrary Dimension by A New Approximate Scheme for The Centrifugal Term, arxiv: 1104.0302v2[quant-ph], 2012.

[17] Y. Xu, S. He and C.S. Jia, Approximate analytical solutions of the Klein-Gordon equation with the Poschl-Teller potential including the centrifugal term, Phys. Scr. 81, 2010, 045001.

[18] A.N. Ikot and L.E. Akpabio, Approximate Solution of Schrodinger Equation with Rosen Morse Potential Including the Centrifugal Term, App. Phys. Res., 2(2), 2010, 202.

[19] N. Rosen and P. M. Morse, On the Vibrations of Poly-atomic Molecules,Physics Review, 42( 2), 1932.

[20] A.N. Ikot, L. E. Akpabio and E.B Umoren, Exact Solution of Schrodinger Equation with Inverted Wood-Saxon and Manning-Rosen Potential, J. Sci. Res 3 (1), 2011, 25-33.

[21] A. S. Halberg, Quasi-Exact Solvability of a Hyperbolic Intermolecular Potential Induced by an Effective Mass Step, Int. J. Maths and Mathematical sciences, DOI10.1155/2011/358198, 2011, 1-9.

[22] H. Goudarzi and V. Vahidi, Supersymmetric Approach for Eckart Potential Using the NU Method, Adv. Studies Theor. Phys. 5(10), $2011,469-476$

[23] S. Meyur and S. Debnath, Solution of the Schrödinger Equation with Hulthén plus Manning-Rosen Potential, Lat. Am. J. Phys. Educ3 (2), 2009, 300-306.

[24] D. Agboola,Dirac equation with spin symmetry for the modified Pöschl-Teller Potential in D dimensions, Pram.J.Phys76 (6), 2011, $875-885$.

[25] C. B. Compean, and M. Kirchbach, The Trigonometric Rosen-Morse Potential in the Supersymmetric Quantum Mechanics and Its Exact Solutions, arXiv: quant-ph/0509055v2, 2005, 1-15.

[26] D.E. Alvarez -Castillo, C. B. Compean, M. Kirchbach, Rotational Symmetry and Degeneracy: A Cotangent-Pertubed Rigid Rotator of Unpertubed Level Multiplicity, arXiv: 1105.1354v1 [quant-ph], 2011, 1-10.

[27] D.E Alvarez -Castillo and M. Kirbach, Exact Spectrum and Wave Functions of The Hyperbolic Scarf Potential in Terms of Finite Romanovski Polynomials,Rev. Mex. Fis.E53 (2), 2007, 143-154.

[28] S.Flugge, Practical Quantum Mechanics(New York: Springer-Verlaag, 1971).

[29] Fis. David Edwin Alvarez Castillo, Exactly Solvable Potentials andRomanovskiPolynomials in Quantum Mechanics, Master Thesis., Universidad Aut'onoma de San Luis Potos'I, Instituto de F'1sica, Mexico, 2007.

[30] J. Derezi'nski and M. Wrochna, Exactly solvable Schrodinger operators, arXiv: 1009.o541v2(math-ph), 2009.

[31] H. Hassan, Y. B. Hoda, L. Liang-Liang, Approximate Analytical Solutions to the Generalized Pöschl-Teller Potential in Dimensions, CHIN. PHYS. LETT29 (2), 2012, 020303. 